It is posted here by permission of transcript Verlag for personal use only, not for redistribution.

\title{
Zur Gegenwart der sprachlich-beruflichen Integration von Flüchtlingen: Chancen und Risiken
}

\author{
IBRAHIM CINDARK, DAVID HÜNLICH
}

\section{EINLEITUNG}

Nicht nur in Bezug auf die aktuelle Einwanderung der Flüchtlinge wird im Kontext der Migration häufig die Frage aufgeworfen, ob solche Prozesse eine Bedrohung oder Bereicherung für die Aufnahmegesellschaft darstellen. Dabei werden nicht selten die Perspektive, die Wünsche und die Hoffnungen der Migranten aus dem Diskurs ausgeklammert. Da jedoch die Migration - und in der Folge die Integration von Migranten - ein wechselseitiger Prozess ist, erachten wir es für essenziell, beide Seiten im Blick zu behalten und von Chancen und Risiken zu sprechen, die solche Entwicklungen sowohl für das Einwanderungsland als auch für die Migranten in sich bergen.

In unserem Aufsatz konzentrieren wir uns auf die Chancen, die sich aus der aktuellen Migration ergeben. Dabei werden wir die Ergebnisse aus unserer Begleitstudie zu einer Qualifizierungsmaßnahme für Flüchtlinge (»Perspektive für Flüchtlinge «, kurz PerFPlus) darlegen, die wir in einer bayerischen Kleinstadt begleitet haben. Solche Maßnahmen können als wichtige Bestandteile der neuen Willkommenskultur in Deutschland betrachtet werden und sind zunächst als eine Chance für beide Seiten zu verstehen. Das Einwanderungsland kann mithilfe solcher Initiativen gezielt für Arbeitsbereiche und Berufsgruppen werben, in denen es an Nachwuchs mangelt. Auf der anderen Seite bieten solche Maßnahmen den Zugewanderten eine Chance, sich in der hiesigen Arbeitswelt zu orientieren und Berufsfelder zu erkunden, die ihnen bislang noch nicht oder anders bekannt waren (so wie die Berufe in den Herkunftsländern ausgeübt werden). 
Maßnahmen wie PerFPlus bergen allerdings auch Risiken: Wenn sie ihr Ziel verfehlen und Frustrationen auf beiden Seiten erzeugen, sind Arbeitslosigkeit, lange Warteschleifen und möglicherweise politische Polarisierung die Folge. Insofern ist eine schnelle Intervention hinsichtlich der Verbesserung solcher Maßnahmen in der gegebenen Situation essenziell. Nachdem wir in den nächsten beiden Abschnitten zunächst auf die aktuelle Fluchtmigration und unser Projekt »Deutsch im Beruf: Die sprachlich-kommunikative Integration von Flüchtlingen« eingehen, analysieren wir im Anschluss ausführlich die Anlage und Durchführung der Maßnahme PerFPlus, die wir ethnografisch begleitet haben. ${ }^{1}$

\section{FLÜChtLINGE IN DEUTSCHLAND}

Die Zahlen darüber, wie viele Geflüchtete 2015 nach Deutschland gekommen sind, schwankten in letzter Zeit zum Teil erheblich. Im Januar 2016 berichtete die Bundesregierung noch, dass es knapp 1,1 Millionen Menschen waren (vgl. BAMF 2016a: 10). Nach Angaben des Bundesamts für Migration und Flüchtlinge (BAMF) wurden aber in diesem Zeitraum `nur 476.649 Asylanträge gestellt (vgl. BAMF 2016a: 11). Die Diskrepanz zwischen diesen beiden Angaben hat mehrere Gründe: Die Zahl von 1,1 Millionen Flüchtlingen geht auf das EASY-

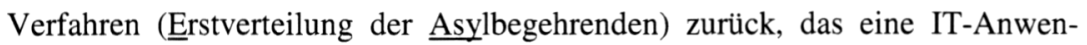
dung zur Erstverteilung der Flüchtlinge auf die einzelnen Bundesländer ist. Da aber nach diesem Verfahren keine persönlichen Daten erfasst werden, sondern lediglich Informationen wie Geschlecht oder Herkunftsland, sind Fehl- und Mehrfacherfassungen nicht ausgeschlossen. Fehlerfassungen kommen zum Beispiel dadurch zustande, dass viele Flüchtlinge Deutschland nur zur Durchreise in andere EU-Länder nutzten. Mehrfach erfasst wurden sie, wenn sie sich statt in das zugewiesene Bundesland auf eigene Faust zu Bekannten oder Verwandten in anderen Bundesländern begaben. Eine letzte Erklärung für die Diskrepanz liefert mit Sicherheit auch die große Zahl der Geflüchteten. Da das BAMF gar nicht über die personellen Kapazitäten verfügte, von allen Flüchtlingen im gleichen Jahr die Asylanträge entgegenzunehmen, ist mit Sicherheit ein Großteil der letztjährigen Flüchtlinge unter den 723.027 Asylanträgen wiederzufinden, die 2016 bis November gestellt wurden (vgl. BAMF 2016b). Im September 2016 wurde

1 An dieser Stelle danken wir herzlich unseren wissenschaftlichen Hilfskräften Kristin Bauer, Oleksandra Gubina, Peter Gyülvészi und Marc Oberle für ihr engagiertes Zuarbeiten für diesen Aufsatz, sowie Rahel Beyer und Ralf Knöbl für ihre wertvollen Korrekturen. 
dann die Zahl der tatsächlich in Deutschland angekommenen Flüchtlinge auch deutlich nach unten korrigiert. Mittlerweile geht die Bundesregierung davon aus, dass 2015 etwa 890.000 Flüchtlinge nach Deutschland kamen, was dennoch eine historische Rekordmarke bedeutet (vgl. Zeit online 2016).

Die Bundesregierung reagierte auf diese »Flüchtlingskrise $\ll^{2}$ mit zwei Gesetzesänderungen, die im Oktober 2015 (Asylpaket I) und März 2016 (Asylpaket II) verabschiedet wurden. Die Reformen verfolgten zusammengefasst drei Ziele (vgl. Bundesregierung 2016: 5-9):

1. Die Gruppe der Flüchtlinge mit guter Bleibeperspektive aus Ländern wie Syrien oder Irak sollen mit den neuen Gesetzen schneller integriert werden: Hierzu zählen Regelungen wie Öffnung der Integrationskurse auch für Flüchtlinge mit einer Aufenthaltsgestattung (Asylpaket II), sicherer Aufenthaltsstatus während der dreijährigen beruflichen Ausbildung oder Änderungen in der Beschäftigungsordnung, sodass Fachkräfte schon nach drei Monaten arbeiten dürfen.

2. Die Gruppe der Flüchtlinge mit schlechter Bleibeperspektive soll schneller abgeschoben werden: Hier hat man Länder wie Afghanistan und Kosovo auf der Liste der sicheren Herkunftsstaaten hinzugefügt und zum Beispiel Abschiebungen ohne vorherige Ankündigungen in die Rechtslage aufgenommen.

3. Durch einige Gesetzesverschärfungen sollen andere Menschen davon abgebracht werden, nach Deutschland kommen zu wollen: So sollen die Asylbewerber Sachleistungen statt Geldzahlungen bekommen und das Recht auf Familiennachzug soll für alle Flüchtlinge, die den geringeren Status des sogenannten subsidiären Schutzes haben, für zwei Jahre ausgesetzt werden.

2 Wie Lehmann (2015: 7) bemerkt, kann man im Zusammenhang mit der großen Fluchtmigration von einem »Krisendiskurs« in den europäischen Ländern sprechen, wobei die deutschsprachigen Medien vor allem den Ausdruck »Flüchtlingskrise« verwenden, während außerhalb von Deutschland meist die Ausdrücke »Migrationskrise« bzw. »Migrantenkrise« gebraucht werden. 


\section{DAS IDS-PROJEKT: "DEUTSCH IM BERUF: DIE SPRACHLICH-KOMMUNIKATIVE INTEGRATION VON FLÜCHTLINGEN «}

Der Leiter des Max-Planck-Instituts zur Erforschung multireligiöser und multiethnischer Gesellschaften, Steven Vertovec, bezeichnet die Fluchtmigration von 2015 als die "zweite Wende« (Vertovec 2015) für Deutschland, die das Land nachhaltig verändern wird. Nach seiner Einschätzung werden die gesellschaftlichen Transformationen dermaßen tiefgreifend sein, dass die Formulierung "seit der Flüchtlingskrise e eine ebenso geläufige Redewendung sein wird wie die Formulierung »seit der Wende«. Um die Migrations- und Integrationsprozesse von Anfang an dokumentieren und analysieren zu können, wurde am Institut für Deutsche Sprache (IDS) zu Beginn des Jahres 2016 das Projekt »Deutsch im Beruf: Die sprachlich-kommunikative Integration von Flüchtlingen « gestartet. ${ }^{3}$ Das Projekt untersucht zum einen in einer ethnografischen Feldstudie, wie der Prozess des Erwerbs der kommunikativen Kompetenzen verläuft, die für erfolgreiche fachliche Kommunikation im Beruf und die interpersonale Integration in Arbeitsteams erforderlich sind. Ein zweiter Bestandteil des Projekts ist die Durchführung einer Sprachstandserhebung in allgemeinen Integrationskursen. In Zusammenarbeit mit dem Goethe-Institut Mannheim werden bei dieser zweistufigen Erhebung die Sprachbiografien und Sprachlernfortschritte von Integrationskursteilnehmern erfasst und analysiert.

Die Sprachstandserhebung wird bundesweit an sieben Standorten in fünf verschiedenen Bundesländern durchgeführt. Die erste Erhebung zu Beginn der allgemeinen Integrationskurse fand von September bis November 2016 statt. In insgesamt 42 Kursen nahmen 607 Menschen an der Erhebung teil, wobei mehr als die Hälfte der Teilnehmer angab, Flüchtling zu sein. ${ }^{4}$ Bei dieser ersten Erhe-

3 Das IDS hat auch früher stets auf die wichtigsten Migrationsprozesse reagiert und deren sprachlich-soziale Erscheinungen unter die Lupe genommen: So wurden in der Vergangenheit das »Gastarbeiterdeutsche« (Keim 1978), die sprachliche Integration von Aussiedlern (Berend 1998; Meng 2001; Reitemeier 2006) und die Kommunikation in verschiedenen Milieus von türkeistämmigen Migranten der zweiten und dritten Generation (Keim 2008; Cindark 2010) untersucht.

4 In der Umfrage haben wir versucht, mit zwei Fragen diesen Personenkreis zu ermitteln: In einer ersten Frage haben wir nach den Gründen der Anwesenheit in Deutschland gefragt, worauf die Teilnehmenden u.a. angeben konnten, aus politischen, religiösen etc. Gründen aus dem Heimatland geflohen zu sein. In einer zweiten Frage haben wir nach ihrem Status in Deutschland gefragt, worauf die Teilnehmenden u.a. mit 
bung geht es darum, Informationen zu Sozialdaten und Sprachbiografien der Teilnehmer einzuholen und zu erfragen, wie viele sprachliche Kontakte sie zur einheimischen Bevölkerung in alltäglichen und beruflichen Kontexten haben. Die zweite Erhebung, die wir am Ende derselben Kurse im Frühjahr und Sommer 2017 durchführen werden, beinhaltet einen Performanztest in Form eines nachgestellten Vorstellungs- bzw. Bewerbungsgespräches. Ziel der Untersuchung ist es, ein Bild davon zu bekommen, wie gut sich die Teilnehmer am Ende der Kurse beruflich selbst darstellen können.

Neben der Sprachstandserhebung führen wir in unserem Projekt ethnografisch fundierte Interaktionsanalysen in Ausbildungskontexten und im Berufsleben durch. In Kooperationen mit mehreren Partnern ${ }^{5}$ untersuchen wir hier, wie gut die sprachliche und soziale Integration der Flüchtlinge in diesen Situationen gelingt und von welchen Faktoren und Bedingungen sie abhängig ist. Im Fokus unserer multimodalen Interaktionsanalysen steht vor allem die Frage, welche sprachlichen und interkulturellen Phänomene sich in Interaktionstypen wie Anleitungs-, Team- und Evaluationsgesprächen als gelungene und nicht-gelungene Praktiken der kommunikativen Integration feststellen lassen. In unserer Ethnografie dokumentieren und analysieren wir zum einen den längsschnittlichen Integrationsverlauf von einzelnen Geflüchteten. Zum anderen begleiten wir einzelne Qualifizierungsmaßnahmen, die wir teilnehmend beobachten und untersuchen. Im nächsten Abschnitt präsentieren wir unsere Einblicke in eine innovative Maßnahme, die in einer bayerischen Kleinstadt mit insgesamt 23 Teilnehmern durchgeführt wurde.

»Flüchtling in Deutschland « antworten konnten. Dementsprechend haben wir bei unserer Erhebung diejenigen als »Flüchtlinge « kategorisiert, die sich bei der letztgenannten Frage diesem Status zuordneten und/oder bei der vorherigen Frage nach Motiven die Flucht als Migrationsgrund nannten.

5 Kooperationspartner sind die Hochschule der Wirtschaft für Management in Mannheim sowie die Agentur für Arbeit Regensburg und lokale Anbieter von Ausbildungsmaßnahmen für Geflüchtete und Partner aus der Wirtschaft und Berufsförderung. 


\section{QuALIFIZIERUngsmassnahmen ALS TEIL DER WILLKOMMENSKULTUR - ethnografische Analyse einer Massnahme}

Deutschland war lange Zeit ein Einwanderungsland wider Willen. Nach dem Zweiten Weltkrieg bis zum Millennium gab es mehrere Migrationsschübe, ohne dass diese Prozesse von politischer Seite wirklich tiefgreifend gestaltet wurden. In den anderthalb Dekaden nach 1945 wurden über 13 Millionen Vertriebene und (deutschstämmige) Flüchtlinge in der Bundesrepublik aufgenommen (vgl. Herbert 2003: 193 ff.; Terkessidis 2000: 16). Daneben begann die Bundesrepublik ab 1955, die damals sogenannten "Gastarbeiter « anzuwerben, die für das deutsche Wirtschaftswunder benötigt wurden. Bis zum Anwerbestopp von ausländischen Arbeitskräften im Jahre 1973 kamen verschiedenen Schätzungen zufolge zwischen 14 und 20 Millionen »Gastarbeiter « nach Deutschland. ${ }^{6}$ Die überwiegende Mehrheit von ihnen kehrte aber wieder in die Heimatländer zurück, sodass 1973 nur noch 2,6 Millionen »Gastarbeiter« in der Bundesrepublik beschäftigt waren (IQ 2003: 199). Ende der 1980er Jahre und zu Beginn der 1990er Jahre waren es schließlich vor allem Spätaussiedler und Asylbewerber, von denen jeweils um die 400.000 jährlich nach Deutschland migrierten. Trotz dieser immensen Migrationen lautete aber das Credo der bundesrepublikanischen Regierungspolitik bis 1998 stets: "Wir sind kein Einwanderungsland.» (Herbert 2003: 262)

Seit den frühen 2000ern begann sich die Politik des Landes jedoch stark zu wandeln und den gesellschaftlichen Realitäten anzupassen: Im Jahre 2000 wurde der Staatsbürgerschaftsparagraf reformiert, 2005 trat das Zuwanderungsgesetz (das zum ersten Mal Integrationskurse für Migranten vorsah!) und 2006 das Allgemeine Gleichbehandlungsgesetz in Kraft. Schließlich begann man seit den 2010ern auch an einer neuen »Willkommenskultur« zu arbeiten, die es Zugewanderten leichter machen soll, sich in Deutschland zu integrieren (IQ 2013: 7). Die oft zitierte Aussage "Wir schaffen das « von Bundeskanzlerin Angela Merkel, die sie im Jahre 2015 als Reaktion auf die Herausforderungen der Fluchtmigration formulierte, kann als Kern dieser neuen, proaktiven Einwanderungspolitik gesehen werden. Wichtige inhaltliche Aspekte der Willkommenskultur sind auf sprachlicher Ebene das Angebot der mehrmonatigen Integrationskurse und im Hinblick auf die berufliche Integration die vielfältigen Qualifizierungsmaß-

6 Seidel-Pielen (1995: 24) und Bade/Oltmer (2005: 73) sprechen von 14 Millionen »Gastarbeitern«, die Deutschland insgesamt anwarb, Oberndörfer (2005: 111) von über 20 Millionen. 
nahmen, die den Migranten angeboten werden. Insofern ist es von zentraler Bedeutung, diese beruflichen Maßnahmen wissenschaftlich zu begleiten und die Frage zu beantworten, wie diese Maßnahmen zum Nutzen aller Beteiligten verbessert werden können.

\section{PerFPlus: Hintergrund und Struktur}

Im Hinblick auf die berufliche Qualifizierung und Vermittlung fallen anerkannte Asylbewerber unter die Zuständigkeit der Jobcenter, Asylbewerber bzw. Geduldete unter die der Bundesagentur für Arbeit (BA). "Perspektive für Flüchtlinge Plus " (PerFPlus) ist eine von vielen Maßnahmen, die im Jahr 2015 von der Regionaldirektion Bayern für Asylbewerber und Geduldete mit einer hohen Bleibewahrscheinlichkeit konzipiert wurden. ${ }^{7}$ Ziel aller Maßnahmen ist es, die Geflüchteten möglichst umfassend und zügig auf eine Arbeitsaufnahme in Deutschland vorzubereiten, indem berufsfachliche Kenntnisse, Fähigkeiten und Fertigkeiten festgestellt und Sprachkenntnisse vermittelt werden. Den rechtlichen Rahmen solcher Maßnahmen bildet $\S 45$ des Sozialgesetzbuches (Drittes Buch). Da die Bundesagentur rechtlich gesehen keine allgemeine Deutschförderung finanzieren darf, beschränkt sich die Vermittlung von Deutschkenntnissen in allen Maßnahmen auf berufsbezogenes Deutsch.

Der wesentliche Vorläufer von PerFPlus, nämlich die Maßnahme »Perspektive für Flüchtlinge« (PerF), bietet Teilnehmern über einen Zeitraum von drei Monaten eine Kompetenzfeststellung sowie Unterstützung bei Bewerbungsvorbereitungen in verschiedenen Berufsfeldern. Auch punktuelle sprachliche Förderung und Praktika in Betrieben sind vorgesehen. Bei der siebenmonatigen Maßnahme PerFPlus werden etliche Bereiche von PerF intensiver gestaltet: Laut der Ankündigung sind 40 Stunden für eine »Standortbestimmung « und »Bedarfsanalyse « und weitere 90 Stunden für eine "Eignungsfeststellung « der Teilnehmer vorgesehen. Dabei sollen der Bildungsstand, der bisherige berufliche Werdegang und die vorhandenen Kenntnisse und Fähigkeiten erfasst und eine Förder- und Integrationsstrategie abgestimmt werden. Es folgen 280 Zeitstunden (ca. 8 Wochen) Kenntnisvermittlung in praktischen Unterrichtseinheiten, die zumeist in Werkstattunterrichten stattfinden. In "tätigkeitsorientierten Erprobungen« von 6 Wochen, d. h. in der Regel in betrieblichen Praktika, soll die Anwendung der

7 Ähnliche Maßnahmen sind zum Beispiel »Brückenjahr 21 plus«, »EQ Flucht«, »Integration durch Arbeit« (IdA) oder "Fit in Arbeit «. Sie unterscheiden sich im Wesentlichen durch die Länge, die Alterszielgruppe und die Ausrichtung auf bestimmte Berufsfelder. 
erlernten Fähigkeiten vertieft werden. Mögliche Felder für Werkstätten und Praktika sind Berufe im Büro und Sekretariat sowie rund um Recht und Verwaltung, Berufe in Handel, Transport, Lager, Logistik, Hotel- und Gaststättengewerbe sowie Berufe in der Versorgung und Installation, Berufe mit Pflanzen (aus dem Berufsfeld Landwirtschaft, Natur, Umwelt), Berufe mit Lebensmitteln (aus dem Berufsfeld Produktion, Fertigung), Gesundheit und Soziales, Elektrotechnik, Metall und das Baugewerbe, Berufe mit Farben und Lacken, mit Holz sowie Bau, Architektur und Vermessung. Einen zentralen Teil nimmt der Sprachunterricht ein: In 365 Zeitstunden (bis zu 10 Wochen) sollen berufsbezogene Deutschkenntnisse erworben werden. Zwei weitere Wochen sind für Bewerbungen und die Vermittlung in Arbeit vorgesehen.

Die wöchentlich 35 Zeitstunden Anwesenheit der Teilnehmer verteilen sich gleichmäßig auf die fünf Wochentage. Während der zeitliche Umfang der Maßnahme und ihrer Teile vorgeschrieben ist, gilt bei der inhaltlichen Durchführung der Maßnahme das Prinzip der Flexibilität. Der Anbieter kann die Inhalte und Module des Kurses im Maßnahmezeitraum verteilen, wie es nach den örtlichen Gegebenheiten am sinnvollsten erscheint. Die genannten Module können sequenziell oder parallel verlaufen. Beim sequenziellen Verlauf werden bei der Kenntnisvermittlung monatsweise verschiedene Arbeitsbereiche >beschnuppert und den Teilnehmern so ein Vergleich diverser Berufsfelder ermöglicht. Ein paralleles Angebot von verschiedenen Werkstätten und Praktika erfordert im Gegensatz dazu wesentlich mehr personelle und räumliche Ressourcen und ist entsprechend schwieriger umzusetzen. Die Gestaltung der Wochenstunden ist ebenfalls flexibel: So kann die Sprachförderung halbtags oder ganztags angeboten werden, sodass sich inhaltlicher Unterricht und Sprachvermittlung entsprechend halbtäglich oder täglich abwechseln.

Die Bundesagentur für Arbeit ist wie alle öffentlichen Auftraggeber gesetzlich verpflichtet, Aufträge zur Deckung ihres Bedarfs grundsätzlich im Rahmen von öffentlichen Ausschreibungen zu vergeben. Über die lokalen Agenturen werden Angebote von lokalen Bildungsanbietern eingeholt und schließlich Verträge über die Durchführung geschlossen.

\section{Analyse der Maßnahme}

Im vorliegenden Fall einer PerFPlus-Maßnahme in einer bayerischen Kleinstadt, die im Juni 2016 begann und im Dezember 2016 endete, wurden wir von der örtlichen Arbeitsagentur der BA als zusätzlicher, begleitender Partner zugelassen, um durch gezieltes Feedback unsererseits die zukünftigen Maßnahmen zu verbessern. Aufgrund der wirtschaftlichen Gegebenheiten im Umkreis der Klein- 
stadt waren für die Kenntnisvermittlung in den Werkstätten des Kurses zunächst die Fachbereiche Metall/Maschinenbau, Bau/Architektur/Vermessung sowie Gesundheit/Soziales/Pädagogik vorgesehen. Die Teilnehmer sollten sequenziell in mehrwöchigen Werkstattunterrichtseinheiten des Anbieters in die Berufsfelder eingeführt und gegen Ende der Maßnahme in betriebliche Praktika vermittelt werden. Im Kurs waren anfangs 20 Teilnehmer. Durch den Ausstieg einiger Teilnehmer und das Nachrücken neuer Teilnehmer nahmen insgesamt 23 Personen teil. ${ }^{8}$ Der Großteil der Teilnehmer stammte aus Syrien, nur drei Teilnehmer kamen aus dem Irak.

In Begleitung der Maßnahme führten wir sowohl Einzel- als auch Gruppeninterviews durch. Interviewpartner waren neben den Teilnehmern auch die Kursleiterin, die Ausbilder in den Fachbereichen, der Anbieter der Maßnahme sowie die Vertreter der Bundesagentur auf lokaler und regionaler Ebene. Wir besuchten einige Teilnehmer auch in ihren Unterkünften und sprachen mit ihren ehrenamtlichen Helfern, um die sozialen Faktoren einordnen zu können, die die Maßnahme indirekt beeinflussen. Als Kernstück unserer Arbeit wurden diverse Ausbildungskontexte per Video und Audio aufgenommen und anschließend analysiert. Wir dokumentierten vor allem die Werkstätten im Bereich Metall und Bau, weil hier Videoaufnahmen möglich waren. ${ }^{9}$ In den folgenden drei Abschnitten präsentieren wir die aus unserer Sicht wichtigsten Analysen und Ergebnisse.

\section{Reihenfolge integrativer Maßnahmen hinsichtlich des Spracherwerbs}

Ein wesentliches Hindernis bei der Durchführung der Maßnahme PerFPlus waren die unzureichenden Sprachkenntnisse der Teilnehmer zu Kursbeginn. Hier stand die ursprüngliche Rechtslage vor Verabschiedung des Asylpaketes II im Widerspruch zu der Konzeption der Maßnahme. Da PerFPlus sich vor allem an Asylbewerber und Geduldete ohne Zugang zum Integrationskurs richtete, konnte von Grundkenntnissen des Deutschen bei den meisten Teilnehmern nicht ausgegangen werden. Bei fast allen Interviews und Gesprächen mit den Teilnehmern

8 Kriegstraumata psychischer und physischer Art sowie die Stimmungslage der Flüchtlinge aufgrund der Geschehnisse in der Heimat spielten im Verlauf der Maßnahme immer wieder eine Rolle, nicht nur beim Ausstieg oder Fernbleiben vereinzelter Teilnehmer. Es ist nicht möglich, in diesem Aufsatz auf dieses komplexe Thema einzugehen.

9 Videodokumentationen in einem Krankenhaus, einer Altenpflegeeinrichtung und einer Praxis für Physiotherapie wurden leider abgelehnt. In einigen Fällen durften wir jedoch Audioaufnahmen machen. 
zu Beginn des Kurses waren wir auf arabischsprachige Dolmetscher angewiesen. Erst gegen Ende der Maßnahme verbesserte sich die kommunikative Situation im Kurs spürbar. Die Kursleiterin stellte im Abschlussinterview den direkten Bezug zwischen sprachlichem Zugang und tieferem Verständnis für die Denkweise der Teilnehmer her: Sie wisse jetzt erst gegen Ende der Maßnahme »wie [die Teilnehmer] denken, weil ich jetzt verstehen kann, was sie sagen«. Für den zukünftigen Vermittlungserfolg von Maßnahmen wie PerFPlus ist daher das sprachliche Einstiegsniveau der Teilnehmer von zentraler Bedeutung. Da sich die derzeitigen Zuständigkeiten vor und nach dem erfolgreichen Abschluss eines Asylverfahrens jedoch unterscheiden und mit der Anerkennung die Zuständigkeit von der lokalen Agentur für Arbeit an das Jobcenter fällt, ist noch unklar, wie der Spracherwerbsprozess in Projekten wie PerFPlus nachhaltiger gestaltet werden soll. Eine engere Kooperation der Ämter beim Entwurf der Angebote wäre eine denkbare Lösung.

\section{Interaktionsanalyse}

Obwohl das kommunikative Grundproblem struktureller Natur ist (PerFPlus vor dem Integrationskurs statt umgekehrt), lohnt es sich, die Praktikumssituationen auf sprachlich-kommunikative Muster hin zu untersuchen, auf die man zukünftig direkter reagieren könnte. Das folgende Beispiel ist einer Werkstattsituation im Metallbereich entnommen. Ein Meister (M) erklärt den beiden syrischen Praktikanten (P1 und P2), wie eine Säulenbohrmaschine zu bedienen ist. ${ }^{10}$

\section{Abbildung 1: Einweisung in den Gebrauch einer Säulenbohrmaschine}

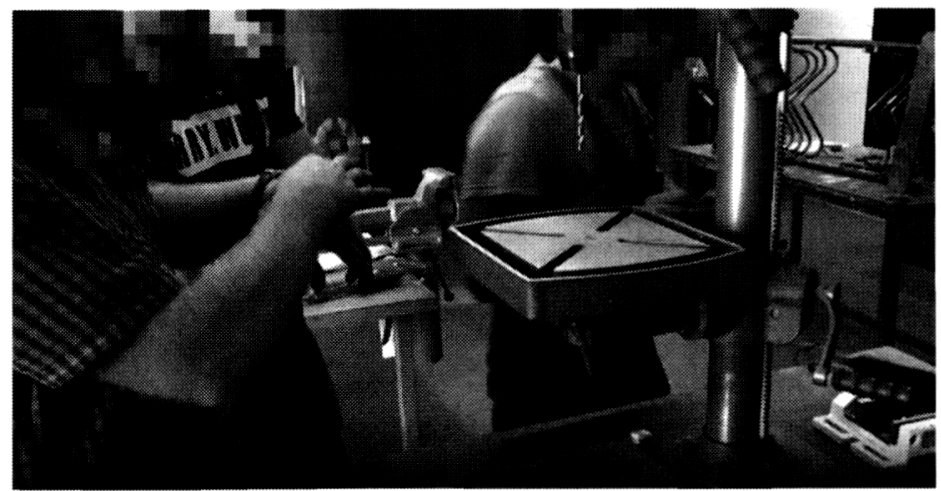

Quelle: IDS (2016) Screenshot aus eigenem Video

10 Transkriptionskonventionen nach GAT2 vgl. Anhang. 


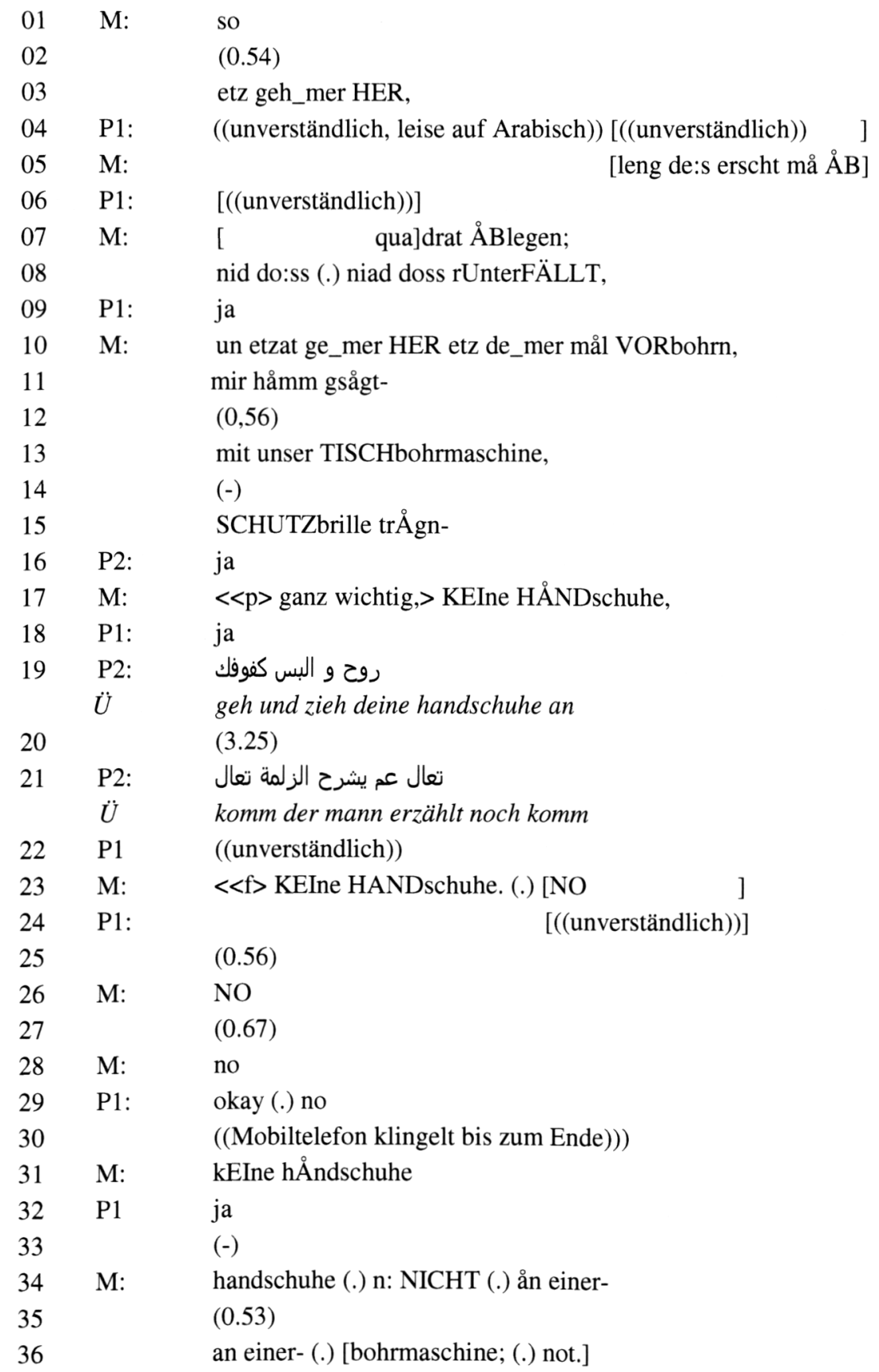


Die hier wiedergegebene Interaktionspassage ist sehr dicht. Der Meister (M) geht mit den beiden Praktikanten (P1 und P2) an eine Tischbohrmaschine und will ihnen erklären, was bei Metallbohrungen zu bedenken ist. Zunächst legt er die Metallplatte, in die gebohrt werden soll, zur Seite und kommentiert dies: »etz geh_mer HER, leng de:s erscht må ÅB, quadrat ÅBlegen; nid do:ss (.) niad doss rUnterFÄLLT, « (Z. 3-8). Im Anschluss geht er auf die wichtigen Sicherheitsaspekte ein, auf die man bei der Arbeit mit Tischbohrmaschinen achten sollte: »mir håmm gsågt- $(0,56)$ mit unser TISCHbohrmaschine, $(0,38)$ SCHUTZbrille trÅgn- $<<$ p > ganz wichtig, $>$ KEIne HÅNDschuhe, « (Z. 11-17). Darauf reagiert der ältere Praktikant (P2), indem er fälschlicherweise - und genau gegenteilig zu dem, was der Meister gesagt hat - den Jüngeren auf Arabisch auffordert, sich die Handschuhe zu holen und sie anzuziehen (Z. 19). P1 kommt dieser Aufforderung nach, dreht sich um und geht zur Werkbank, wo sich die Handschuhe befinden. Da aber in dieser Zeit (Z. 20) P2 bemerkt, dass der Meister mit halb offenem Mund und etwas irritiert das Geschehen verfolgt, fordert er P1 auf, doch nochmal zurückzukommen: »komm der mann erzählt noch komm« (Z. 21). Jetzt interveniert auch der Meister, der inzwischen realisiert hat, dass die syrischen Praktikanten die Negation sprachlich nicht verstanden haben: »KEIne HANDschuhe (.) NO (0.56) NO (0.67) no« (Z. 23-28). Mit seinem Codeswitching ins Englische und dem mehrmaligen Wiederholen des »No signalisiert er an dieser Stelle die enorme Bedeutung dieser Sicherheitsmaßnahme an der Bohrmaschine. Die Sequenz wird damit beendet, dass der jüngere Praktikant seinem älteren Kollegen auf Arabisch vorwirft, nur das gemacht zu haben, was dieser ihm aufgetragen hat: »ich hab auf dich gehört« (Z.37).

Folgende Aspekte sind konkret bei dieser Interaktion - aber allgemein in sehr vielen anderen von uns beobachteten Kontexten in den Qualifizierungsmaßnahmen - von großer Bedeutung:

- die Spracherwerbssituation generell: Die beiden syrischen Praktikanten waren zum Aufnahmezeitpunkt erst seit einigen Monaten in Deutschland und hatten noch keinen Integrations- bzw. Sprachkurs absolviert, weshalb sie nur über rudimentäre Deutschkenntnisse verfügten. Der deutsche Ausbilder scheint aber - vielleicht aufgrund der Kursziele von PerFPlus - davon auszugehen, dass sie mehr Deutsch verstehen, als sie sprechen. Für diese Interpretation spricht: Obwohl es bei dieser Interaktion unter anderem um sehr wichtige Sicherheitsmaßnahmen am Arbeitsplatz geht, stellt der Meister an keiner Stelle 
verständnissichernde Rückfragen. Er interagiert mit den Praktikanten sprachlich so, wie wenn er mit deutschsprachigen Auszubildenden interagieren würde. Erst in Z. 20, als die Missverständnisse offensichtlich werden, verändert er seine Sprechweise.

- Rückfragen zur Verständnissicherung fehlen aber auch auf Seiten der Geflüchteten: Ob die beiden Praktikanten in der hier analysierten Passage aus kulturellen und/oder sprachlichen Gründen auf Rückfragen verzichten, kann nicht mit Sicherheit geklärt werden. Es kann sein, dass die Interaktion zwischen Lehrer/Ausbilder und Schüler kulturell bedingt für syrische und irakische Teilnehmer mit anderen Erwartungen verbunden ist als im deutschsprachigen Kontext. Zumindest wurde uns von Praktikumsleitern auch in späteren Phasen der Maßnahme, in denen die Teilnehmer bereits etwas mehr Deutsch konnten, vom höflichen Schweigen der Teilnehmer berichtet, was aus deutscher Sicht durchaus auch als Passivität interpretiert werden könne. Eine wesentliche Aufgabe für unsere weiteren Analysen wird sein, hier die adäquaten Antworten zu finden, ohne in die »Kultur-erklärt-alles-Falle« zu tappen.

- das regional geprägte Deutsch des Ausbilders, das Codeshifting bzw. die Codefluktuation zwischen Oberpfälzisch und hochdeutscher/fachsprachlicher Lexik: In den bairischen Dialekten werden Personalpronomen, die dem Verb direkt folgen, fast immer enklitisiert und phonetisch assimiliert. So wird im Oberpfälzischen die Kombination aus dem Verb < gehen> und <wir> (dialektale Nebentonform [mə]) zu »ge_mer« [gemə] und <tun wir> (dialektal vollformig [doun]) zu »de_mer« [demə]. Der Kontrast zu standardnahen Verschleifungen und anderen Regionalvarianten mag für den Muttersprachler nicht erheblich sein, kontrastiert aber für Sprachlerner des Hochdeutschen stark mit dem Sprachgebrauch im Klassenzimmer bzw. Integrationskurs. Noch drastischer als in der Metallwerkstatt war das Problem für die Praktikanten im Pflegebereich. Sie empfanden das Dialektverstehen für die Kommunikation mit alten oder geschwächten Patienten und Gesprächspartnern aus dem ländlichen Raum als absolut essenziell.

Diesen Abschnitt zur Sequenzanalyse zusammenfassend kann man hier zum Schluss die Frage stellen, inwiefern standardferne Varianten, die fester Bestandteil des Berufslebens in den südlichen Bundesländern und allgemein in ländlichen Gebieten sind, nicht auch Teil der sprachlichen Vorbereitung auf Berufskommunikation sein müssten. Ebenso könnte man auch in das Curriculum der Integrationskurse relativ unkompliziert ein Dialektmodul integrieren. In der Schweiz sind Module zur Dialektvermittlung an Migranten zum Beispiel sehr erfolgreich. 
Des Weiteren sollten nach unseren ersten Beobachtungen optimalerweise alle Praktikumsleiter und -anbieter zumindest in Form einer Kurzschulung für die wichtigsten Aspekte der interkulturellen Kommunikation und des Deutscherwerbs am Arbeitsplatz sensibilisiert werden.

\section{Passgenauigkeit der Maßnahme}

Es zeigte sich im Verlauf der Maßnahme, dass die sequenzielle Durchführung der Kenntnisvermittlung in den Werkstätten für Metall, Holz und Pflege beachtlichen Schwierigkeiten gegenüberstand, auf die die Kursleiterin eingehen und flexibel reagieren musste. Beim Abschlussinterview stellte sie fest, dass die praktischen Angebote in den Werkstätten in den meisten Fällen nicht den beruflichen Neigungen der Teilnehmer entsprachen. Unsere Beobachtungen bestätigen, dass die meisten Teilnehmer bereits über Arbeitserfahrung verfügten und den angebotenen Werkstätten kritisch bis ablehnend gegenüberstanden. In Absprache mit der Arbeitsagentur begann die Kursleiterin nach alternativen Praktika zu suchen - zunächst für einzelne und schließlich für fast alle Teilnehmer. Außerplanmäßige Praktika wurden zum Beispiel in der Gastronomie, in einem Friseursalon, in einem Reinigungsbetrieb, bei einem Fliesenleger, in einer KfzWerkstatt und in einer Konditorei angeboten. Einen ähnlich individualisierten Ansatz, der die Neigungen der Teilnehmer direkter anspricht, schlägt die Kursleiterin auch für zukünftige PerFPlus-Maßnahmen vor.

Es muss festgehalten werden, dass die Maßnahme in ihrer Ursprungsform ohne die flexible Reaktion der lokalen Arbeitsagentur, des Anbieters und der Kursleiterin ihr Ziel nicht erreicht hätte. Berücksichtigt man jedoch das Engagement des Anbieters und der Kursleiterin, kann dank der Eingriffe trotzdem von einem Erfolg der Maßnahme gesprochen werden. Ein Sankey-Diagramm, das die Situation der 23 Teilnehmer (mit Nachrückern und Frühaussteigern) ca. einen Monat vor Ende des Kurses abbildet, unterstreicht die Wirkung, die der Eingriff der Kursleitung hatte. 
Abbildung 2: Die Situation aller Kursteilnehmer ca. einen Monat vor Ende von PerFPlus

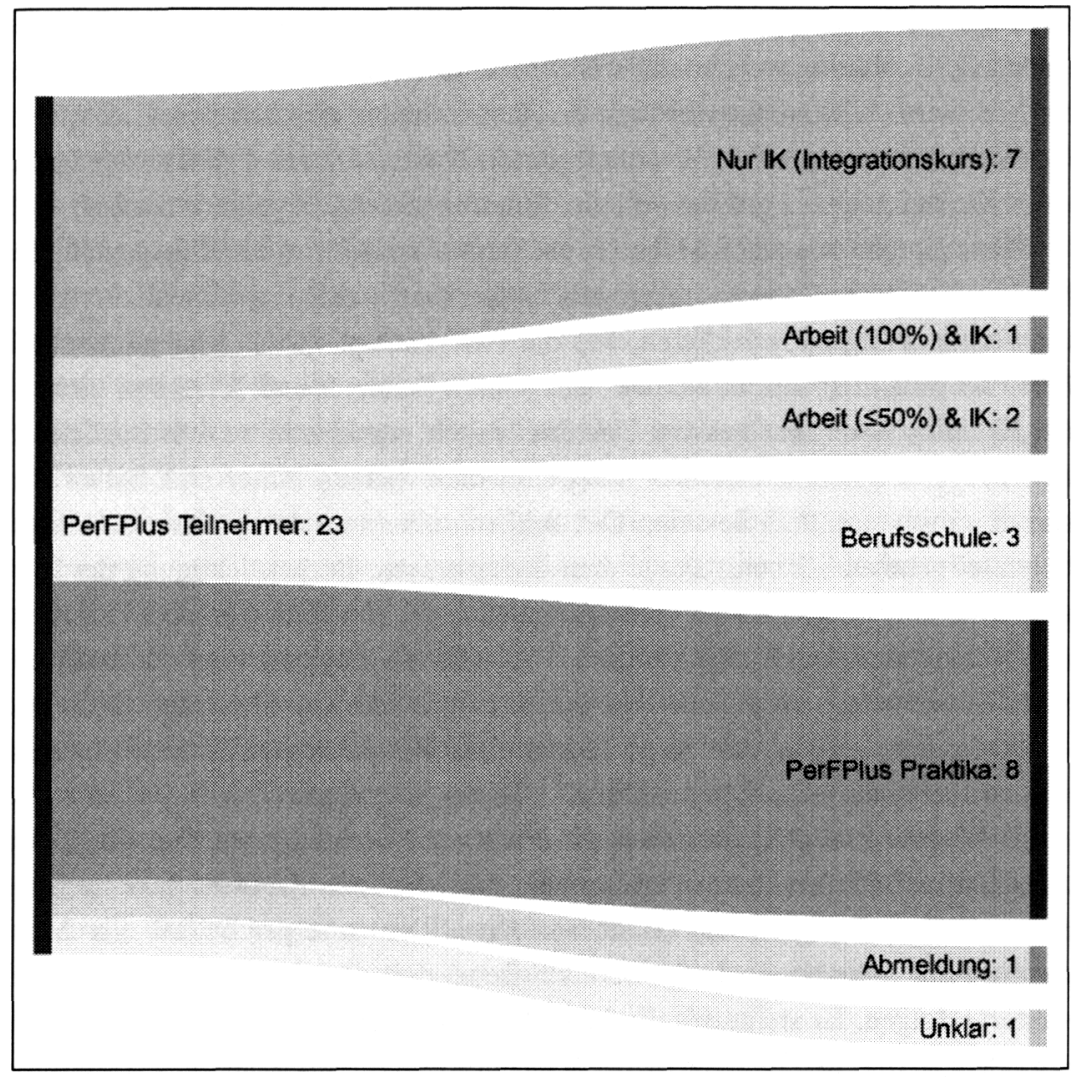

Quelle: Eigene Darstellung

In dreizehn Fällen kümmerte sich die Kursleiterin nach und vor den individualisierten Praktika aktiv um die Übermittlung in situationsgerechtere Alternativen. Zehn Teilnehmern wurde vor Kursende der Übergang in einen Integrationskurs (IK) ermöglicht. Dies ist der normale, vorgezeichnete Weg für alle anerkannten Asylbewerber in Deutschland. Das Besondere hier ist allerdings, dass drei IKTeilnehmer auf Grund von PerFPlus zukünftig auch berufstätig sind - einer sogar in Vollzeit. Drei weitere Kursteilnehmer wurden von lokalen Berufsschulen übernommen. Auch dies ist ein Ergebnis der Teilnahme an PerFPlus, weil die Geflüchteten von der Kursleiterin gecoacht und begleitet wurden. Dank des Eingriffs der Kursleiterin hatte also die Hälfte der Teilnehmer bereits einen Monat vor Ende von PerFPlus eine Perspektive für die kommenden Monate. Acht Teil- 
nehmer (in dunkelblau) verblieben noch in der Maßnahme, um die praktischen Angebote (Praktika und Werkstattunterricht) wahrzunehmen. Das Diagramm stellt nicht dar, dass diese Gruppe nach der Maßnahme im Dezember 2017 auch in den IK wechselte und ein weiterer Teilnehmer in Vollzeit beschäftigt wurde. Nur in einem Fall (in orange) wurde der Kurs komplett abgebrochen. Hier spielte die Wohnsituation der Familie eine Rolle. In einem weiteren Fall war der Status eines Kursteilnehmers unklar, weil das Anhörungsverfahren negativ verlief.

Dass die überwiegende Mehrheit der Teilnehmer durch das Engagement der lokalen Kräfte im Integrationsprozess unterstützt wurde, ist erfreulich. Allerdings ist es nötig zu reflektieren, wie die Passgenauigkeit von Maßnahmen wie PerFPlus zukünftig erhöht werden kann, damit Anbieter und Kursleiter nicht in die Situation kommen, massive Umverteilungen vornehmen zu müssen. Sollten zukünftige Kursteilnehmer den Integrationskurs bereits hinter sich haben und dadurch mit wesentlich besseren Deutschkenntnissen an den praktischen Angeboten teilnehmen können, könnte man durch bessere Berücksichtigung der Hintergründe und Neigungen der Teilnehmer auch die Vermittlungsquote in Arbeit, Ausbildung und langfristige Bildungsmaßnahmen erhöhen. Fünf Fallbeispiele von Teilnehmern, deren Deutschkenntnisse über dem Gesamtniveau lagen und denen die Kursleiterin aktiv zu passenden Praktika und weiterführenden Integrationsmaßnahmen verhalf, verdeutlichen dieses.

Die ersten Praktika, die außerhalb des Kurses vermittelt wurden, gingen an zwei Kursteilnehmer, die der Kursleitung früh deutlich machten, dass sie nicht mehr in der Metallwerkstatt arbeiten wollten. Der erste der beiden war Mitte zwanzig und hatte bereits in Syrien als Friseur gearbeitet. Diesen Beruf wollte er weiterverfolgen. Es stellte für ihn kein Problem dar, dass ihn ein Arbeitsumfeld erwarten würde, das sich stark von dem in Syrien unterschied: Im Friseursalon arbeitete er ausschließlich mit weiblichen Kolleginnen zusammen und bediente vorwiegend Frauen als Kunden. Beides war in seiner Heimatstadt für einen männlichen Friseur unüblich. Der zweite Teilnehmer, der in seiner Heimat Geschäftsführer eines Supermarkts war, äußerte den Wunsch, ein Praktikum als Barkeeper zu absolvieren, was ihm auch ermöglicht werden konnte. An der Bar stand er in kontinuierlichem Austausch mit deutschen Muttersprachlern. Interessanterweise führte der Ausstieg der beiden Teilnehmer aus der Werkstatt nicht dazu, dass sie sich von den anderen Kursteilnehmern isolierten. Im Gegenteil: Viele folgten ihrem Beispiel und bemühten sich um eine Ausgliederung aus dem Metallkurs. Der Friseur und der angehende Barkeeper gaben uns nach nur wenigen Wochen ein Interview in rudimentärem Deutsch, in dem sie quasi als Advokaten der restlichen Kursteilnehmer den Deutschunterricht kritisierten und sich über die Fokussierung des Unterrichts auf drei Kursteilnehmer mit akademi- 
schem Hintergrund beklagten. Es muss der proaktiven und direkten Art der beiden angerechnet werden, dass ihnen gegen Ende der Maßnahme ein Ausbildungsplatz (nach dem IK) und eine Arbeitsstelle (während des IK) angeboten wurden. Wäre die Kursleiterin nicht auf die Forderungen der beiden eingegangen und hätte die Teilnehmer stattdessen zu Aktivitäten angehalten, die nicht ihren Neigungen entsprechen (aber im Kursprogramm vorgesehen waren), wäre PerFPlus in diesen beiden Fällen nicht zum Erfolg geworden.

Das proaktive, direkte Kommunizieren - und auch die manchmal damit verbundenen Konflikte - führten auch im Falle der drei akademisch vorgebildeten Teilnehmer, die laut anderer Kursteilnehmer oft die Aufmerksamkeit im Deutschunterricht für sich beanspruchten, zu individualisierten Lösungen. Dabei handelte es sich um drei junge Männer im Alter von Anfang zwanzig Jahren, die als Nachrücker in den Kurs gekommen waren. In der Woche vor Eintritt in die Maßnahme interviewten wir die drei mit Hilfe einer Dolmetscherin. In Syrien hatten zwei von ihnen eine universitäre Ausbildung zum Physiotherapeuten abgeschlossen. Durch PerFPlus stand ihnen nun die Teilnahme an einem Praktikum der Physiotherapie in einem Krankenhaus und einer Praxis in Aussicht. Der dritte Teilnehmer hatte sein Studium der Architektur in Syrien aufgrund des Krieges abgebrochen und kam in der Hoffnung nach Deutschland, es hier beenden zu können. Den drei Teilnehmern war allerdings nicht klar, dass die Praktika im Krankenhaus erst gegen Ende der ansonsten sequenziell verlaufenden Maßnahme geplant waren. Mit dem Einstieg in die Maßnahme in der Metallwerkstatt hatten die drei nicht gerechnet. Bereits nach dem ersten Tag waren sich die drei Teilnehmer sicher, dass es sich bei PerFPlus um eine "schlechte Maßnahme" handele, und verweigerten am zweiten Tag ihre Teilnahme.

Dieses Verhalten stieß wiederum bei den Ehrenamtlichen, die sich intensiv um die Aufnahme der drei Nachrücker in die Maßnahme bemüht hatten, auf Unverständnis und bewirkte eine nachhaltige Enttäuschung. Sehr wahrscheinlich hatten sprachliche Missverständnisse und fehlende Informationen die Kommunikation zwischen Arbeitsagentur, Ehrenamtlichen und den drei Teilnehmern beeinträchtigt. Die drei Männer rechtfertigten ihre Reaktion dann auch durch mehrere Argumente: Zum einen schrieben sie sich bessere Deutschkenntnisse zu als den anderen Teilnehmern und sahen sich bzgl. ihres Deutschniveaus falsch eingeordnet. Dies konnten wir nur teilweise bestätigen. Eher unterschied sich die Lerngeschwindigkeit der drei wohl deutlich von den anderen Teilnehmern. So empfanden sie den Unterricht oft als langweilig, während der Rest des Kurses sich durch die Geschwindigkeit der drei neuen Teilnehmer überfordert fühlte. Mit relativ klaren beruflichen Zielen, nämlich in der Physiotherapie und der Architektur, ähnelte die Grundeinstellung der Teilnehmer auch den Praktikanten im 
Friseursalon und der Bar: Die Arbeit in einer Metallwerkstatt war für sie nicht interessant. Gerne nahmen sie für ein Erreichen der beruflichen Ziele auch eigene Anstrengungen und Kosten in Kauf. So kontaktierte der Architekturstudent etwa kurzerhand selbst einen Architekturprofessor an einer nahe gelegenen Hochschule, besuchte ihn mit der Bahn und meldete sich zu einem fachlichen Vergleichbarkeitstest an, um bisherige Studienleistungen übertragen zu können. Im Praktikum zeigten die Physiotherapeuten keine Berührungsängste zu weiblichen Patienten und unterstellten sich ohne Probleme der Supervision von Frauen. ${ }^{11}$ Wie im Falle des Friseurs kommen solche Konstellationen in der Heimatstadt der beiden nicht vor.

Der Bildungshintergrund dieser Teilnehmer muss als Hauptunterscheidungsmerkmal zu den anderen Teilnehmern gesehen werden. Nach der Unterredung mit den Ehrenamtlichen beschlossen die drei Nachrücker, wieder am Kursprogramm teilzunehmen. Die Teilnahme verlief aber nicht konfliktfrei, sodass sich die Kursleiterin schließlich um die Überführung der drei in ein höheres Modul eines Integrationskurses bemühte. Rechtlich bestand diese Möglichkeit nach der Verabschiedung des Asylpaketes II, obwohl eine Aufenthaltserlaubnis noch nicht bei allen drei vorlag. Obgleich die Teilnehmer nun offiziell aus der PerFPlus-Maßnahme ausgegliedert waren, sorgte die Kursleiterin dafür, dass die ehemaligen Teilnehmer ihr versprochenes Praktikum in einem Krankenhaus und einer Praxis für Physiotherapie antreten konnten. Außerordentliche Flexibilität auf der Seite des Anbieters führte somit zum relativen Erfolg der Maßnahme für diese akademisch vorgebildeten Teilnehmer.

\section{Schluss}

Die auf nationaler Ebene vor einigen Jahren formulierte »Willkommenskultur « muss sich derzeit auf lokaler Ebene in zahlreichen Maßnahmen zur beruflichen Eingliederung bewähren. Die hohe Anzahl neuer Flüchtlinge vor allem aus dem Jahr 2015, aber auch eine Reihe von Berufsfeldern mit Nachwuchsproblemen, machen das Gelingen dieser Maßnahmen zu einer umso dringlicheren Aufgabe. Hier liegen Chancen und Risiken für den Erfolg von Integration. Im vorliegenden Aufsatz wurden unsere Beobachtungen zur Maßnahme PerFPlus vorgestellt und diskutiert. Ein Grundproblem lag in der Diskrepanz zwischen der Ausgangs-

11 Eine ehrenamtliche Helferin hatte dies auch als Problem vorausgesagt, weil einer der drei jungen Männer, die alle muslimischen Glaubens sind, Frauen zur Begrüßung nicht die Hand reiche. Wir wurden nicht Zeuge einer solchen Situation. 
situation der Teilnehmer im sprachlichen und im beruflichen Sinne und den Vorgaben der Maßnahme auf struktureller und inhaltlicher Ebene. Zwar machten alle Teilnehmer deutliche Fortschritte im Deutschen. Gegen Ende der Maßnahme war es uns sogar möglich, einzelne Interviews auf Deutsch zu führen. Grundsätzlich sollte der erste Spracherwerb aber dem Erwerb von Fachsprache vorgeschaltet werden. Situationen, in denen die erste Orientierung im Praktikum an grundlegenden sprachlichen Hindernissen scheitert, können Frustration auf beiden Seiten erzeugen. Auf der Mikroebene der Interaktion zwischen Ausbildern und Teilnehmern zeigen unsere Beobachtungen, dass die Lehrkräfte in den praktischen Fächern eine bessere Vorbereitung auf das Sprachniveau ihrer Teilnehmer benötigen, damit sie den Spracherwerb aktiv unterstützen können. Eine zusätzliche Überlegung wäre es, die Praktikumsanteile im späteren Verlauf des Kurses auch gemeinsam mit deutschen Teilnehmern durchzuführen, um den Migranten die Möglichkeit zu geben, selbst mehr mit Muttersprachlern zu interagieren. So könnten kulturelle Unterschiede leichter überbrückt werden als in einer Lernsituation, in der der deutsche Ausbilder mit den Flüchtlingen alleine interagiert.

Bezüglich der Passgenauigkeit der Maßnahme waren im vorliegenden Fall massive Eingriffe in den Kursverlauf nötig, weil die Interessenlage etlicher Teilnehmer von den sequenziellen Werkstattangeboten abwich. Viele Teilnehmer hatten bereits eine Ausbildung absolviert oder verfügten über Arbeitserfahrungen in einem Feld außerhalb des Angebotes. Ein generelles Problem, das wir bei der beruflichen Integration auch in anderen Maßnahmen und Programmen beobachten, ist die Kompatibilität zwischen den Bedürfnissen der lokalen Wirtschaft und den Interessen der berufserfahrenen Flüchtlinge. Würden berufliche Interessen und Erfahrungen bereits bei der Verteilung der Flüchtlinge in unterschiedliche Wirtschaftsräume berücksichtigt, könnten solche Probleme abgemildert werden. Maßnahmen würden sich dann auch an homogenere Zielgruppen richten. In der Praxis würde dies bedeuten, dass jede Region in Deutschland Einwanderungsziele formuliert und eine erste Standortbestimmung der Teilnehmer nicht erst in den Maßnahmen stattfindet, sondern bereits bei der Verteilung der Migranten in Deutschland. 


\section{ANHANG: EINIGE DER TRANSKRIPTIONSKONVENTIONEN NACH GAT2 ${ }^{12}$}

\section{$\underline{\text { Sequenzielle Struktur/Verlaufsstruktur }}$}

[ ]

Überlappungen und Simultansprechen

[ ]

$\underline{\text { Pausen }}$

(.) Mikropause, geschätzt, bis ca. 0.2 Sek. Dauer

(-) kurze geschätzte Pause von ca. 0.2-0.5 Sek. Dauer

(0.5) gemessene Pausen in Sekunden; Angabe mit einer Stelle hinter dem Punkt

Sonstige segmentale Konventionen

\begin{tabular}{|c|c|}
\hline $\begin{array}{l}\text { und_äh } \\
\text { ((hustet)) }\end{array}$ & $\begin{array}{l}\text { Verschleifungen innerhalb von Einheiten } \\
\text { para- und außersprachliche Handlungen u. Ereignis- } \\
\text { se }\end{array}$ \\
\hline$<<$ hustend $>>$ & $\begin{array}{l}\text { sprachbegleitende para- und außersprachliche Hand- } \\
\text { lungen und }\end{array}$ \\
\hline
\end{tabular}

Ereignisse mit Reichweite

$\begin{array}{ll}\text { : } & \text { Dehnung, Längung, um ca. 0.2-0.5 Sek. } \\ . . & \text { Dehnung, Längung, um ca. } 0.5-0.8 \mathrm{Sek}\end{array}$

Tonhöhenbewegung am Ende von Intonationsphrasen

?

hoch steigend

mittel steigend

,

gleichbleibend

; $\quad$ mittel fallend

. $\quad$ tief fallend

Akzentuierung

$\begin{array}{ll}\text { akZENT } & \text { Fokusakzent } \\ \text { akzEnt } & \text { Nebenakzent }\end{array}$

12 GAT2 steht für »Gesprächsanalytisches Transkriptionssystem 2« (vgl. auch Selting et al. 2009). 
Lautstärke- und Sprechgeschwindigkeitsveränderungen mit Extension

$\begin{array}{ll}<<\mathrm{f}>> & \text { forte, laut } \\ <<\mathrm{p}>> & \text { piano, leise }\end{array}$

Wiedergabe des Dialekts

A bzw. å verdumpftes »a«, nahe am offenen »0« [0]

\section{LITERATUR}

Bade, Klaus/Oltmer, Jochen (2005): »Einwanderung in Deutschland seit dem Zweiten Weltkrieg«, in: Kölnischer Kunstverein et al. (Hg.), Projekt Migration - Ausstellungskatalog, Köln: DuMont, S. 72-81.

BAMF (2016a): Das Bundesamt in Zahlen 2015. Asyl, Migration und Integration. Siehe https://www.bamf.de/SharedDocs/Anlagen/DE/Publikatio nen/Broschueren/bundesamt-in-zahlen-2015.pdf?__blob=publicationFile vom 07.03.2017.

BAMF (2016b): Aktuelle Zahlen zu Asyl. Ausgabe 11/2016. Siehe http://www.bamf.de/SharedDocs/Anlagen/DE/Downloads/Infothek/Statistik/ Asyl/aktuelle-zahlen-zu-asyl-november-2016.pdf?__blob=publicationFile vom 07.03.2017.

Berend, Nina (1998): Sprachliche Anpassung. Eine soziolinguistisch-dialektologische Untersuchung zum Rußlanddeutschen. Tübingen: Narr.

Bundesregierung (2016): Herausforderungen annehmen - Frieden, Freiheit und Wohlstand sichern. Jahresbericht der Bundesregierung 2014/15. Siehe https://www.bundesregierung.de/Content/DE/_Anlagen/2015/12/201512-22-Jahresbericht-2014-2015.pdf?__blob=publicationFile \&v=5 vom 07.03.2017.

Cindark, Ibrahim (2010): Migration, Sprache und Rassismus. Der kommunikative Sozialstil der Mannheimer »Unmündigen« als Fallanalyse für die »emanzipatorischen Migranten«. Tübingen: Narr.

Herbert, Ulrich (2003): Geschichte der Ausländerpolitik in Deutschland. Saisonarbeiter, Zwangsarbeiter, Gastarbeiter, Flüchtlinge, Bonn: Bundeszentrale für politische Bildung $(\mathrm{BpB})$.

IQ (Netzwerk Integration durch Qualifizierung) (2013): Willkommenskultur (und Anerkennungskultur): Hintergrund, Diskussion und Handlungsemfehlungen. Siehe http://digital.zlb.de/viewer/content?action=application\& sourcepath=15744013/FS_DiM_Arbeitspapier_Willkommen_2013.pdf\&for mat=pdf vom 07.03.2017 
Keim, Inken (1978): Gastarbeiterdeutsch. Untersuchungen zum sprachlichen Verhalten türkischer Gastarbeiter, Tübingen: Narr.

Keim, Inken (2008): Die »türkischen Powergirls« - Lebenswelt und kommunikativer Stil einer Migrantinnengruppe in Mannheim. Tübingen: Narr.

Lehmann, Julian (2015): »Flucht in die Krise - Ein Rückblick auf die EU->Flüchtlingskrise ««, in: Aus Politik und Zeitgeschichte Jg. 65, 52 S. 7-11.

Meng, Katharina (2001): Russlanddeutsche Sprachbiografien. Untersuchungen zur sprachlichen Integration von Aussiedlerfamilien. Tübingen: Narr.

Oberndörfer, Dietmar (2005): "Zuwanderung, kulturelle Vielfalt und Integration im demokratischen Verfassungsstaat «, in: Karl-Heinz Meier-Braun/Reinhold Weber (Hg.), Kulturelle Vielfalt. Baden-Württemberg als Einwanderungsland, Stuttgart: Kohlhammer, S. 110-125.

Reitemeier, Ulrich (2006): Aussiedler treffen auf Einheimische. Paradoxien der interaktiven Identitätsarbeit und Vorenthaltung der Marginalitätszuschreibung in Situationen zwischen Aussiedlern und Binnendeutschen. Tübingen: Narr.

Seidel-Pielen, Eberhard (1995): Unsere Türken. Annäherung an ein gespaltenes Verhältnis. Berlin: Elefanten Press.

Selting, Margret et al. (2009): »Gesprächsanalytisches Transkriptionssystem 2 (GAT 2)«, in: Gesprächsforschung - Online-Zeitschrift zur verbalen Interaktion 10, S. 353-402. Siehe http://www.gespraechsforschung-online.de/file admin/dateien/heft2009/px-gat2.pdf vom 07.03.2017.

Terkessidis, Mark (2000): Migranten. Hamburg: Rotbuch.

Vertovec, Steven (2015): »Was die Zuwanderung mit Deutschland macht «, in Süddeutsche Zeitung vom 20.10.2015. Siehe http://www.sueddeutsche.de/po litik/fluechtlinge-was-die-zuwanderung-mit-deutschland-macht-1.2710550 vom 06.03.2017.

ZEIT ONLINE (2016): »2015 kamen weniger Flüchtlinge als gedacht«, in: ZEIT ONLINE vom 30.09.2016. Siehe http://www.zeit.de/politik/deutsch land/2016-09/2015-kamen-890-000-asylsuchende-nach-deutschland vom 07.03.2017. 